\title{
Cryo-Electron Microscopy Analysis of a 105 kDa Protein Particle: The Xylulose Kinase from $E$. Coli
}

\author{
Hui-Ting Chou, Eric di Luccio, David Wilson*, Henning Stahlberg* \\ Molecular and Cellular Biology, University of California, Davis, CA 95616, USA. \\ *corresponding authors: dave@alanine.ucdavis.edu and HStahlberg@ucdavis.edu
}

Xylose is the second most abundant carbohydrate in terrestrial plants. The interest in metabolic engineering for the pathway of the fermentation from xylose to ethanol has been high for the past two decades because it implies the possibility of utilizing agricultural waste to produce ethanol as fuel [1]. The prime choice for the fermentation in industrial bioethanol production is Saccharomyces cerevisiae combined with heterologous gene expression encoding nonoxidative xylose phosphate pathway from other yeast species, such as Pichia stipitis [2]. In the process of xylose fermentation, the ATP-dependent phosphorylation of D-xylulose catalyzed by xylulose kinase (XK) is the ratelimiting step. The structure of the $52.6 \mathrm{kDa} E$. coli XK was recently determined by X-ray crystallography (E. di Luccio et al., submitted). Here, we applied cryo-electron microscopy and single particle structure reconstruction techniques to determine the low-resolution structure of the non-crystallized vitrified protein. The data show dimeric XK particles that correspond to a mass of 105.2 $\mathrm{kDa}$. Such particles are at the lower detection limit of the single particle cryoEM method.

Purified XK sample at a concentration of $1 \mathrm{mg} / \mathrm{ml}$ was vitrified on holey carbon film grids (Quantifoil) and imaged at liquid nitrogen temperature in a JEOL JEM-2100FEG transmission electron microscope, operated at 200kV ([3], Fig. 1A). A total 487 particle images were chosen from 26 micrographs and subjected to computer image processing using the SPIDER software [4]. Particles were centered, classified (Fig. 1B), re-aligned onto the class averages and a 3D structure was reconstructed by backprojection of the class averages. In an angular assignment refinement process, particles were then iteratively re-assigned by multi-reference alignment to projections of the volume. The final reconstruction is shown in Fig. 2A,C. Its resolution was determined by Fourier shell correlation [5] at $36 \AA$ ( 0.5 criterion), which was limited by the low molecular weight of the protein particles, and possibly also the flexibility of the interface between the two xylulose kinase monomers.

Due to the small particle size, the alignment of the raw particle images onto given references in SPIDER failed, so that the class-averages with the improved signal-to-noise ratio were used instead. The X-ray structure was not used during the entire image processing except for the determination of the handedness of the final reconstruction.

Cryo-electron microscopy of single particles has a lower detection limit for the protein particle size. This is due to decreased electron scattering from smaller particles, which results in less information obtained from the protein and therefore less accurate alignment of the noisy images. The size and particle number of the xylulose kinase dimer imaged here is close to Henderson limit for phasecontrast electron microscopy [6]. Despite the low symmetry (two-fold) of the XK particles, alignment and classification could be carried out, and the improved signal-to-noise ratio of the classaverages was sufficient for a $3 \mathrm{D}$ reconstruction. 


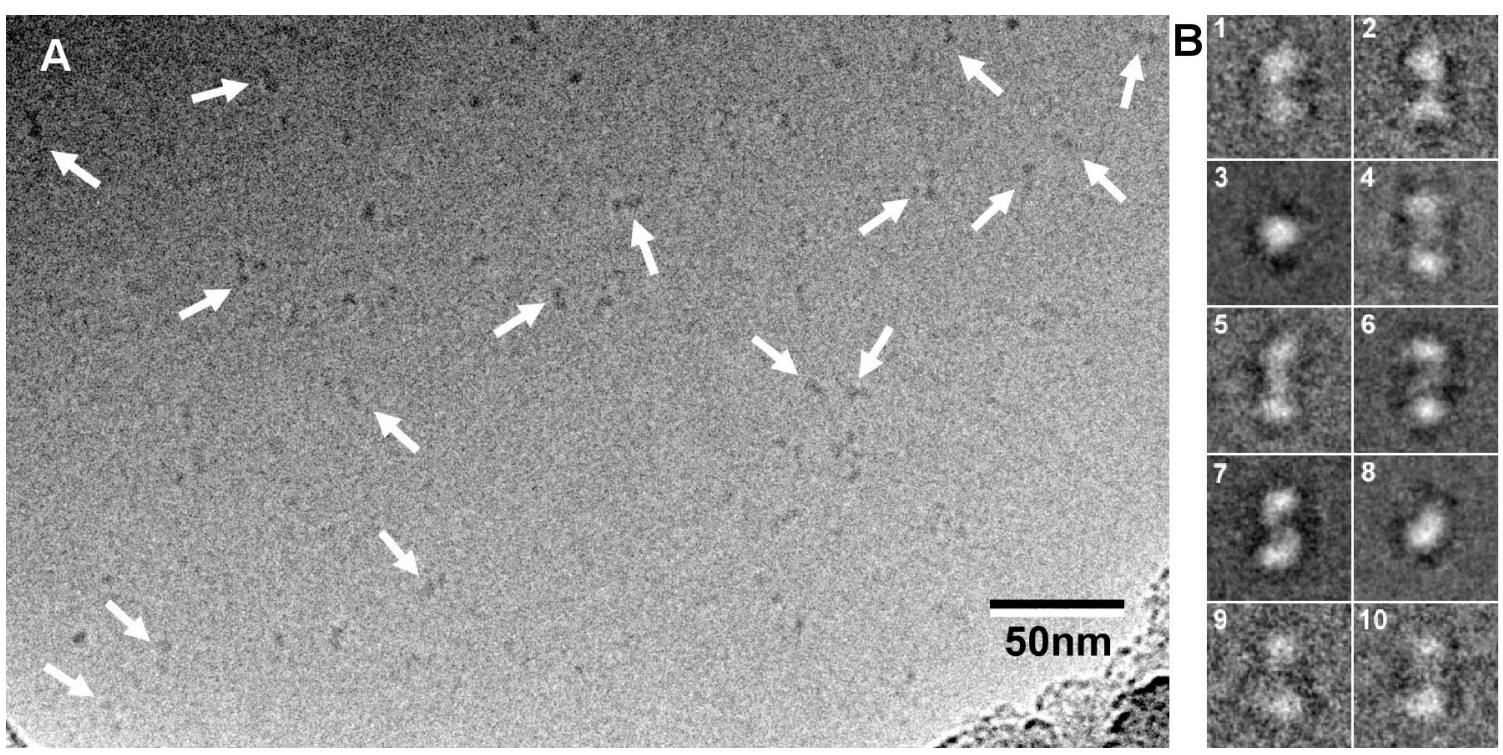

Figure 1. (A) A cryo-EM image of xylulose kinase particles in vitrified buffer solution. The arrows indicate dimeric xyluose kinase particles. (B) Some class averages. The panels have a width of 19.2 $\mathrm{nm}$.
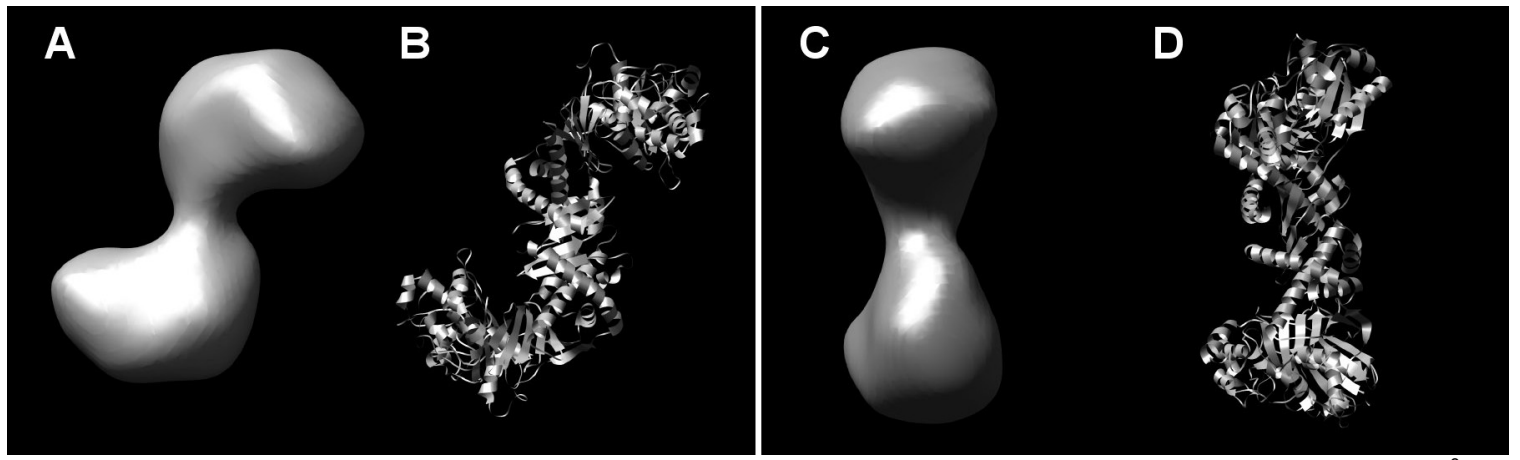

Figure 2. Two views of the surface-rendered density map from cryo-EM limited to $36 \AA$ resolution $(\mathrm{A}$ and $\mathrm{C})$ and the atomic model $(\mathrm{B}$ and $\mathrm{D})$ from X-ray crystallography of xylulose kinase.

\section{References}

[1] T.W. Jeffries et al., Appl Microbiol Biotechnol 63 (2004), 495.

[2] K. Karhumaa et al, Yeast 22 (2005), 359.

[3] J. Dubochet et al., Quart Rev Biophys 21 (1988), 129.

[4] J. Frank et al., J Struct Biol, 116 (1996), 190.

[5] M.J. van Heel, Ultramicroscopy, 21 (1987), 100.

[6] R. Henderson, Ultramicroscopy, 28 (1992), 171. 